\title{
Treatment with transfemoral bare-metal stent of residual aortic arch dissection after surgical repair of acute type an aortic dissection
}

\author{
Luigi Di Tommaso, Raffaele Giordano, Ettorino Di Tommaso, Giusi Di Palo, Gabriele Iannelli \\ Adult and Pediatric Cardiac Surgery, Department of Advanced Biomedical Sciences, University of Naples Federico II, Napoli, Italy \\ Contributions: (I) Conception and design: L Di Tommaso, R Giordano; (II) Administrative support: L Di Tommaso, G Iannelli; (III) Provision of \\ study materials or patients: L Di Tommaso; (IV) Collection and assembly of data: E Di Tommaso, G Di Palo; (V) Data analysis and interpretation: L \\ Di Tommaso, R Giordano; (VI) Manuscript writing: All authors; (VII) Final approval of manuscript: All authors. \\ Correspondence to: Raffaele Giordano, MD. Via Pansini 5, 80131 Naples (Na), Italy. Email: r.giordano81@libero.it.
}

Background: Here we evaluate the usefulness of transfemoral uncovered stent implantation to avoid secondary conventional surgery for residual type A aortic dissection (TAAD) of the aortic arch after ascending aorta replacement.

Methods: From June 2009 to April 2015, 11 patients were treated with transfemoral implantation of uncovered stents in the aortic arch after surgical replacement of ascending aorta performed on average $4.7 \pm 2.3$ years earlier. An enlarged dissected aortic arch or a dangerous median growth of more than $5 \mathrm{~mm} / \mathrm{yr}$ or impending rupture presenting as chest pain were indications for treatment. The dissected aortic tracts diameter must not exceed $45 \mathrm{~mm}$. Five patients (45.5\%) were treated with Djumbodies Dissection System, 6 patients $(54.5 \%)$ with Jotec E-XL aortic stent.

Results: There were no perioperative deaths or permanent neurologic complications. Primary procedural success was obtained in all patients and the residual TAAD in aortic arch was obliterated, with disappearance of the false lumen. Median intensive care unit (ICU) stay was 24 hours; post-operative hospital stay was $5.2 \pm 1.4$ days. One death, not aortic related, occurred during follow-up period (mean $5.2 \pm 1.9$ years). Descending thoracic aorta diameter significantly increased in 3 patients (27.3\%): one patient (9.0\%) needed a secondary conventional surgery, the other $2(18.2 \%)$ of a distal extension with PETTICOAT approach.

Conclusions: Endovascular approach with uncovered metal bare stent is surely an evolving strategy to perform a purely endovascular treatment, indicated only for treatment of an aortic arch with a diameter of less than 40 or $45 \mathrm{~mm}$, to avoid progressive thoracic aortic dilatation and/or rupture.

Keywords: Type A aortic dissection (TAAD); endovascular treatment; uncovered stents

Submitted May 03, 2018. Accepted for publication Oct 15, 2018.

doi: $10.21037 /$ jtd.2018.10.51

View this article at: http://dx.doi.org/10.21037/jtd.2018.10.51

\section{Introduction}

Although recent substantial advances in medical care, acute type A aortic dissection (TAAD) remains one of the most life-threatening cardiovascular disease with a high natural morbidity and mortality rate, of about $50 \%$ in the first 24 hours if the patients remain untreated (1-3). The treatment continues to be challenging, though emergency surgical approach is the only option to improve the survival.
Ascending aorta with hemiarch replacement is the first step in the treatment of these patients (1,3). Mortality reported in recent series from international registry of acute aortic dissection (IRAD) still ranges from $17 \%$ to $26 \%(1,3,4)$.

Unfortunately, surviving operation does not guarantee freedom from subsequent aortic events especially if the intimal tear cannot be removed leaving a patent false lumen leading to progressive descending aortic dilatation and 


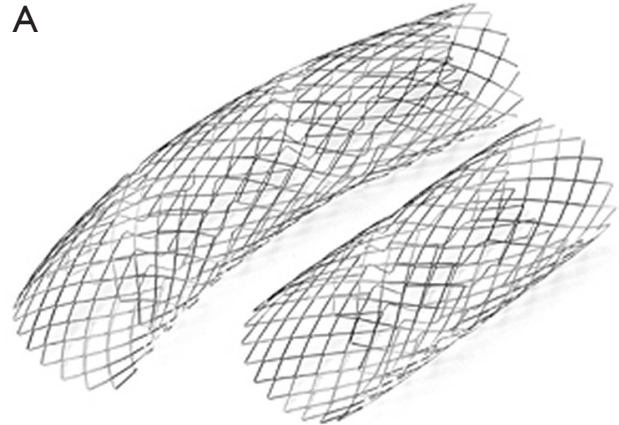

B

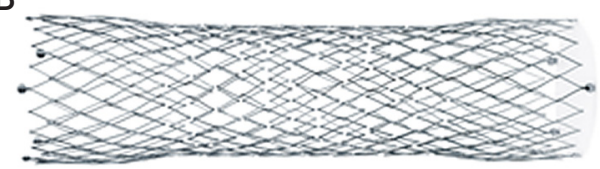

Figure 1 Two types of bare stents implanted. (A) Djumbodies Dissection System; (B) Jotec E-XL stent, reported from illustrative brochure of the Saint Come Chirurgie, and Jotec respectively.

rupture (5). So, these patients can develop a progressive enlargement of aortic arch and distal aorta with a high risk of aortic rupture and need for a secondary aortic surgery (5-7) joined to a higher morbidity and mortality rate than primary surgery (6).

Numerous attempts have been made to achieve a purely endovascular treatment of residual TAAD. Despite these efforts, only few isolated case reports of selected patients with poor clinical impact have been reported in literature (8).

Alternatively, purely endovascular approach with transfemoral implantation of bare stents has emerged as a valid option to conventional surgery in the management of residual TAAD after ascending aorta replacement.

The rationale of these devices is to readapt the dissecting membrane to the adventitial layer in direct continuity to the ascending or aortic hemiarch prosthesis to promote the false lume thrombosis.

In order to evaluate usefulness of these uncovered stents, here we report our non-randomized retrospectively study showing early and mid-term results in particular regarding to late aortic events and false lumen status, after totally percutaneous (transfemoral) endovascular treatment by bare-stents in aortic arch with or without covered stent graft placement in the descending thoracic aorta.

\section{Methods}

From June 2009 to April 2015, 11 patients with residual
TAAD of the aortic arch and downstream aorta, persisting after conventional ascending aorta replacement with graft, were treated with transfemoral deployment of uncovered bare stents in aortic arch, in order to join the dissected layers avoiding collateral branches obstruction.

An enlarged dissected aortic arch beginning from the distal anastomosis of previously shown

surgically implanted aortic graft or a dangerous median growth of the aortic arch diameter of more than $5 \mathrm{~mm} / \mathrm{yr}$ or impending rupture presenting as a recurrent chest pain were indications for treatment. The dissected aortic tracts diameter must not exceed $45 \mathrm{~mm}$.

All patients, informed of the risks of conventional open surgery, refused surgical treatment and provided written informed consent for the procedure.

Preoperatively all patients, in order to assess suitability for the procedure and to determine the size of the implanting stent underwent contrast-enhanced computed tomography (CT) scan of entire aorta and conventional coronary angiography.

Our follow-up program includes serial CT-scan at 1, 6, 12 months, and yearly thereafter for all patients to monitor the aortic disease progression. Patients were seen annually by a cardiac surgeon of our teams. Missing information was completed by contacting the referring patient's physician and/or his or her family. The follow-up was closed on June 2017 and was $100 \%$ complete.

The retrospective study protocol was reviewed and approved by the institutional local ethics committee with protocol no. 189/2016. The study complied with the Helsinki declaration. We obtained personal data treatment consent from all patients. The data were collected in a computerized database.

Two types of bare stent have been implanted: the Djumbodies ${ }^{\circledR}$ Dissection System (DDS) (Saint Come Chirurgie, Marseille, France) (Figure 1A) and Jotec E-XL aortic stent (JOTEC GmbH, Hechingen, Germany) (Figure 1B). The first one is an open stainless-steel balloonexpandable system that can be expanded up to $45-50 \mathrm{~mm}$ pre-mounted on a low pressure ( 0.3 bars) balloon catheter. It has 3 available lengths: 40,90 and $140 \mathrm{~mm}$. It is specifically designed and approved for both type A or B aortic dissections, not suited for aneurysms, that can be used in conjunction with the classic replacement with a graft of the ascending aorta. The mesh is sufficiently large to bring together the dissected layers without occluding main vital branches such as supra-aortic arteries in the aortic arch, renal and mesenteric arteries in the abdomen. 


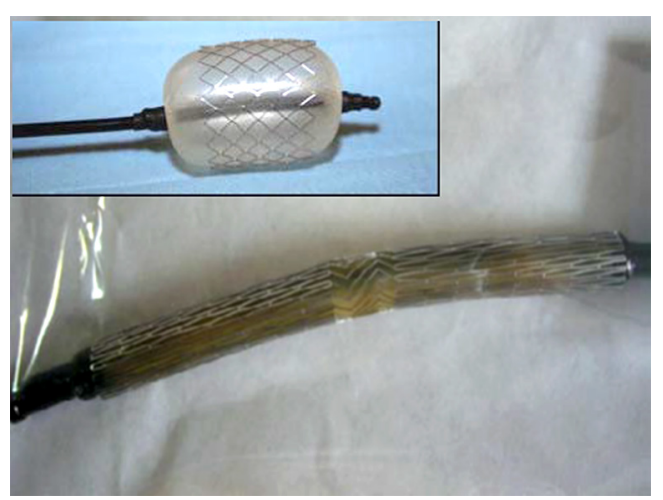

Figure 2 Djumbodies Dissection System with pre-mounted balloon, reported from illustrative brochure of the Saint Come Chirurgie.

On the contrary the Jotec E-XL aortic stent is a nitinol self-expandable stent especially designed for the treatment of lesions, as aortic dissection or stenosis, of the aortic arch and/or of the descending and abdominal aorta. It can be expanded up to $40 \mathrm{~mm}$, the closed-cell structure on both ends of the unique stent design provides optimal vessel fixation and avoidance of stent migration. Ideal flexibility and therefore ideal conformability to the vessel anatomy is guaranteed by the open-cell structure in the middle.

Study endpoints included operative (30-day) and midterm mortality from any cause, overall mortality from aortic causes, late aortic event-free survival and the need for further aortic or vascular re-interventions. Sudden unexplained deaths were included in aortic-related mortality.

\section{Statistical analysis}

Continuous data are presented as mean \pm standard deviation (SD) and categorical data as proportion as appropriate. Fisher exact test (for sparse data) was used to compare categorical variables. Unpaired Student $t$-test was used to compare independent continuous variables. Two-tailed tests of significance are reported, and $\mathrm{P}$ values less than 0.05 are considered statistically significant. Kaplan-Meier analysis was used to study patient survival and event-free status, with the log-rank test (Cox-Mantel) to ascertain differences between the groups. Data were analyzed by SPSS version 14.0 for Windows (SPSS, Inc., Chicago, Illinois, USA).

\section{Operative technique}

The procedures were performed by a combined team of cardiac surgeons and cardiologists, in a hybrid operating room equipped with a portable digital C-arm fluoroscopy OEC 9900 (GE Healthcare, Chalfont St Giles, UK). The Djumbodies Dissection System deployment was performed under general anesthesia and mechanical ventilation while local anesthesia was used for Jotec E-XL stent implantation. Blood pressure was monitored via radial artery. Brain oxygenation was monitored using a near-infrared spectroscopy.

In all cases, vascular access was achieved by surgical exposure of the right or left common femoral artery, systemic heparin $(70 \mathrm{U} / \mathrm{kg})$ was routinely administrated to obtain an activated clotting time longer than 200 seconds. A super-stiff 300-cm-long 0.035-inch Back-up Meier wire (Boston Scientific, Boston, USA), introduced through the femoral vascular access was advanced under fluoroscopy, through a $6 \mathrm{~F}$ pigtail catheter (Cordis, Hamburg, Germany), at the aortic valve level. In order to set the markers of the proximal landing zone to overlap the implanted ascending aortic graft, intraoperative angiography was performed with a $6 \mathrm{~F}$ pigtail catheter introduced through contralateral femoral artery.

As previously described (9), Djumbodies Dissection System was introduced through an 85 -cm delivery system (LeMaitre Vascular, Inc., Burlington, MA, USA) loaded over a super-stiff guidewire up to the distal end of the conventional graft in the ascending aorta. The stent was introduced into the previously surgically implanted graft for about $2 \mathrm{~cm}$ to overlap the distal border. When a systolic arterial pressure of less than $70 \mathrm{mmHg}$ was reached or pharmacologically (by sodium nitroprusside) or with rapid pacing, the pre-mounted balloon catheter was inflated under fluoroscopy for less than 30 seconds in order to obtain a no-flow in the supra-aortic vessels for a very short time (Figure 2). This procedure was repeated whether a second or third stent implant was necessary for complete extension across the entire aortic arch toward the descending thoracic aorta to obtain in some instances a bridge with a covered stent-graft distally to left subclavian artery (LSA).

Instead self-expanding Jotec E-XL aortic stent, loaded onto the super-stiff guide wire to the level of the proximal aortic arch was released under fluoroscopy overlapping the graft previously implanted. 
Table 1 Patient preoperative characteristics

\begin{tabular}{lc}
\hline Variable & No. of patients $(\mathrm{n}=11)(\%)$ \\
\hline Age (years) & $60.5 \pm 8.2$ \\
Male sex & $9(81.8)$ \\
Preoperative status & \\
Hypertension & $11(100.0)$ \\
Hyperlipidemia & $8(72.7)$ \\
Diabetes mellitus & $6(54.5)$ \\
COPD & $9(81.8)$ \\
Coronary artery disease & $3(27.3)$ \\
Cerebrovascular disease & $2(18.2)$ \\
Chronic renal insufficiency & $2(18.2)$ \\
Connective tissue disease & 0 \\
Previous cardiac/aortic surgery & $11(100.0)$ \\
Time free from previous surgery (yrs) & $4.7 \pm 2.3$ \\
ASA class III & $8(72.7)$ \\
ASA class IV & $3(27.3)$ \\
Thoracic pain & $6(54.5)$ \\
Mntreatable hypertension & $4(36.4)$ \\
\hline Critical preoperative status & 0 \\
\hline
\end{tabular}

COPD, chronic obstructive pulmonary disease; ASA, American Society of Anesthesiologists.

Table 2 Operative data

\begin{tabular}{lc}
\hline Variable & No. of patients $(\mathrm{n}=11)(\%)$ \\
\hline Emergency procedure & 0 \\
General anesthesia & $6(54.5)$ \\
Common femoral access & $11(100.0)$ \\
Stent implantation & \\
Djumbodis Dissection System & $5(45.5)$ \\
Jotec E-XL & $6(54.5)$ \\
Rapid pacing for stent placement & $4(36.4)$ \\
Intentional LSA coverage & $5(45.5)$ \\
Covered stent graft implant & $5(45.5)$ \\
\hline
\end{tabular}

LSA, left subclavian artery.
When associated endovascular stent grafting of the descending thoracic aorta was necessary the delivery system was loaded onto the super stiff guide wire to the level of the thoracic aorta and released under fluoroscopy as previously described (10).

Subsequent aortography was performed to confirm the treatment adequacy, attested by a satisfactory aortic reshaping with a significant exclusion of the residual dissection and patency of cerebral and visceral vessels.

\section{Results}

Out of the 11 patients with residual dissection of the aortic arch, who underwent transfemoral implantation of metal bare stent after ascending aorta replacement, 6 patients $(54.5 \%)$ presented chest pain, 3 patients $(27.3 \%)$ associated with untreatable hypertension. In treated patients, the average diameter of all the aortic arch, including true and false lumen, was $44.1 \pm 2.3 \mathrm{~mm}$. Five patients $(45.5 \%)$ were treated with Djumbodies Dissection System (DDS group) from May 2009 to June 2011, whereas 6 patients (54.5\%) up to april 2015 were treated with Jotec E-XL aortic stent (Jotec $\mathrm{E}-\mathrm{XL}$ group). The stents are sized with respect to the aortic arch diameter and proximal descending thoracic aorta, with an oversize of $10 \%$ with respect of the distal landing zone. In all patients we covered, by bare-stents, the entire aortic arch with the origin of the epiaortic vessels, from the ascending aortic graft to descending thoracic aorta.

Surgical replacement of the ascending aorta had been performed on average $4.7 \pm 2.3$ years earlier. Patient preoperative and operative data are shown in Tables 1 and 2 respectively. Seven patients $(63.6 \%)$ had general anesthesia, all patients of the DDS group and 2 patients of the Jotec E-XL group. The other four patients (36.4\%) had local anesthesia with a conscious sedation. No patient underwent emergency surgery. All procedures were performed after an accurate diagnostic process and a carefully discussed planning for the endovascular treatment

Early postoperative results are shown in Table 3. There were no perioperative deaths or surgical conversion. Primary procedural success was obtained in all patients. In all cases, the residual TAAD in aortic arch was obliterated, with satisfactory arch recontouring and complete disappearance of the false lumen as documented by intraoperative angiography.

The patients of the DDS group received a mean of $2.3 \pm 0.8$ stents of various length in order to cover the entire aortic arch up to the descending aorta. All patients treated 
Table 3 Early results

\begin{tabular}{lc}
\hline Variable & No. of patients $(\mathrm{n}=11)(\%)$ \\
\hline Hospital mortality (30 days) & 0 \\
Surgical conversion & 0 \\
Aortic rupture & 0 \\
Misplacement of bare stent & 0 \\
Prolonged mechanical ventilation & $2(18.2)$ \\
New onset neurologic dysfunction & \\
Temporary & $1(9.0)$ \\
Permanent & 0 \\
Vascular complications & $1(9.0)$ \\
New renal insufficiency & $1(9.0)$ \\
Cardiac complications & 0 \\
ICU stay, days (median) & 1 \\
Hospital stay, (days) & $5.2 \pm 1.4$ \\
\hline
\end{tabular}

ICU, intensive care unit.

with Jotec E-XL had only one stent implantation.

There were no permanent neurologic complications or paraplegia despite entire descending aorta was covered from LSA up to the celiac axis in 8 patients $(72.7 \%)$ with intentional coverage of LSA origin, without revascularization, in 5 patients $(45.5 \%)$. A ventricular fibrillation, quickly converted in absence of sequelae, occurred in one patient (9.0\%) of the DDS group during the balloon inflation time. One patient $(9.0 \%)$ of the Jotec group, without carotid diseases or LSA overstenting showed a transient neurological complication.

One patient $(9.0 \%)$ of the Jotec group showed a vascular injury due to severe calcified atherosclerosis requiring a common femoral artery repair. One patient of the DDS group showed a new renal insufficiency not requiring dialysis. There were no statistically significant differences between the two groups in the incidence of postoperative complications. Two patients of the DDS group and 3 patients of the Jotec group showed early postoperative complications $(\mathrm{P}=0.9)$

Median intensive care unit (ICU) stay was 24 hours (range from 1 to 3 days) with a mean of $1.3 \pm 0.6$ days. Postoperative hospital stay was $5.2 \pm 1.4$ days, less than 5 days, for 5 patients $(45.5 \%)$.

All patients were discharged under medical therapy including antiplatelet (aspirin $100 \mathrm{mg} / \mathrm{d}$ ) and antihypertensive agents. One-month follow-up CT scan confirmed exclusion of the false lumen in the short axis view and a very satisfying remodeling of the arch and descending thoracic aorta in the multiplanar and 3D volume rendering reconstructions (Figures 3,4).

Mean follow-up was $5.2 \pm 1.9$ (range from 2.2 to 8.3 ) years. Late results are shown in Table 4. One death, not aortic related, occurred during follow-up period.

At mid-term follow-up, there were no changes with regard to true lumen diameter at the discharge as well as total diameter of the aortic arch on the CT scans in all patients. Distal aortic false lumen was patency in 6 patients $(54.5 \%)$ and patent false lumen was partially or completely closed in the remainder 4 patients (Table 4).

Descending thoracic aorta diameter significantly increased in 3 patients (27.3\%). Two of these showed at follow-up a distal type I endoleak. One patient (9.0\%), with distal type 1 endoleak, needed a secondary conventional surgery, in a center with high volume, because of distal enlargement of the thoraco-abdominal aorta. The other 2 (18.2\%) patients underwent distal extension of the descending thoracic aortic endoprosthesis with uncovered bare metal stents to restore and support true lumen with PETTICOAT (Provisional Extension to Induce Complete Attachment After Stent-Graft Placement in Type B Aortic Dissection) approach (9). There was no complication. At follow-up there is no further aortic enlargement. A fourth patient showed an early distal aortic enlargement that stopped at subsequent follow-up. Thus, we choice to follow him with a close follow-up.

\section{Discussion}

The treatment of acute TAAD continues to be challenging. Unfortunately, surviving the operation does not guarantee freedom from subsequent aortic events. In many cases the distal intimal tear cannot be removed, and the false lumen frequently remains patent, leading to progressive descending aortic dilatation and rupture (5). Thus, initial emergency surgical treatment is only the first step for these patients $(3,11,12)$, who often develop progressive dilatation of a residual false lumen of aortic arch and downstream aorta with a high risk of aortic rupture (11). Many studies have identified patent false lumen as a risk factor for aortic dilatation and/or rupture (5,6,12-15). Leontyev and coworkers (13) in their study were able to show that a true lumen less than $25 \%$ combined with an aortic diameter greater than $40 \mathrm{~mm}$ on the postoperative CT scan was an 

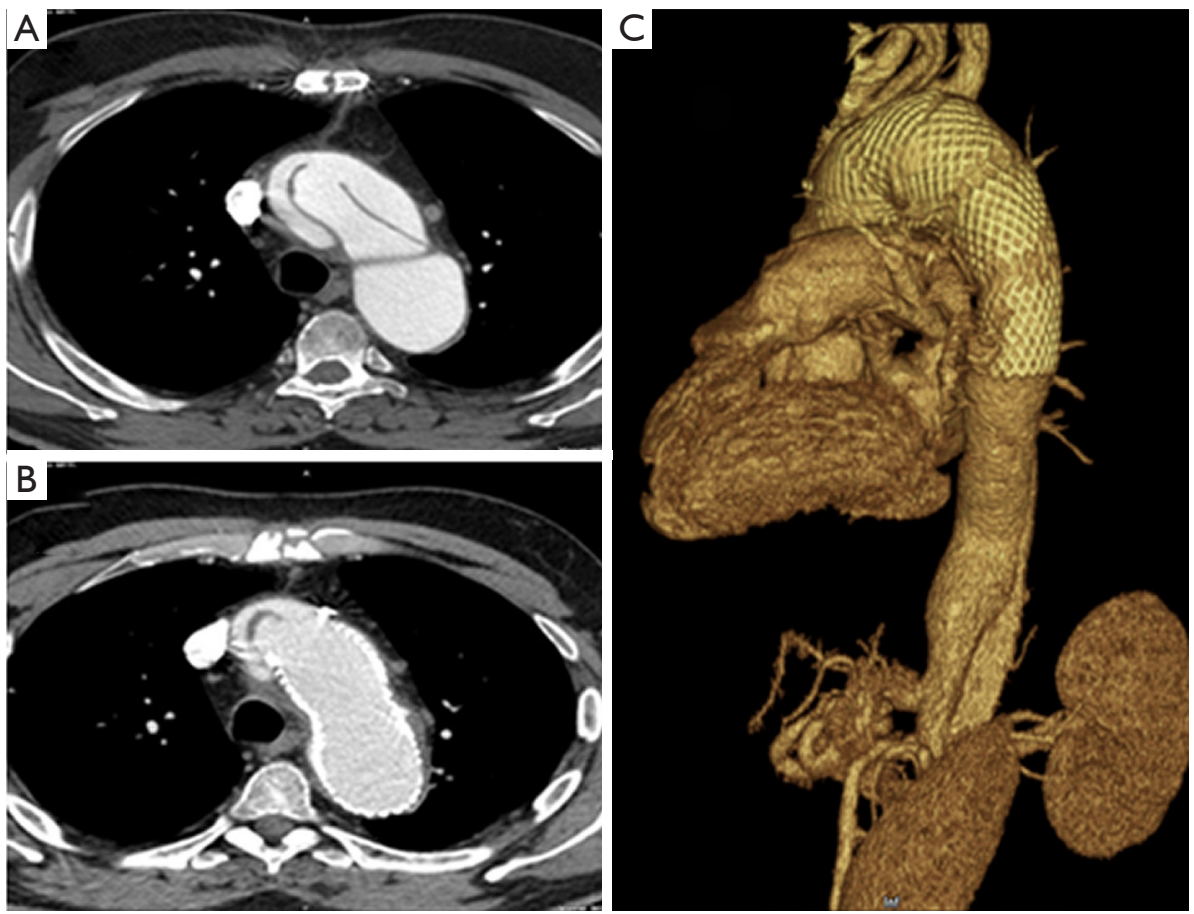

Figure 3 Patient control after treatment of aortic arch by CT scan. (A) Preoperative CT scan; (B) postoperative CT scan; (C) 3D volume rendering reconstruction of implanted Djumbodies Dissection System. CT, computed tomography.
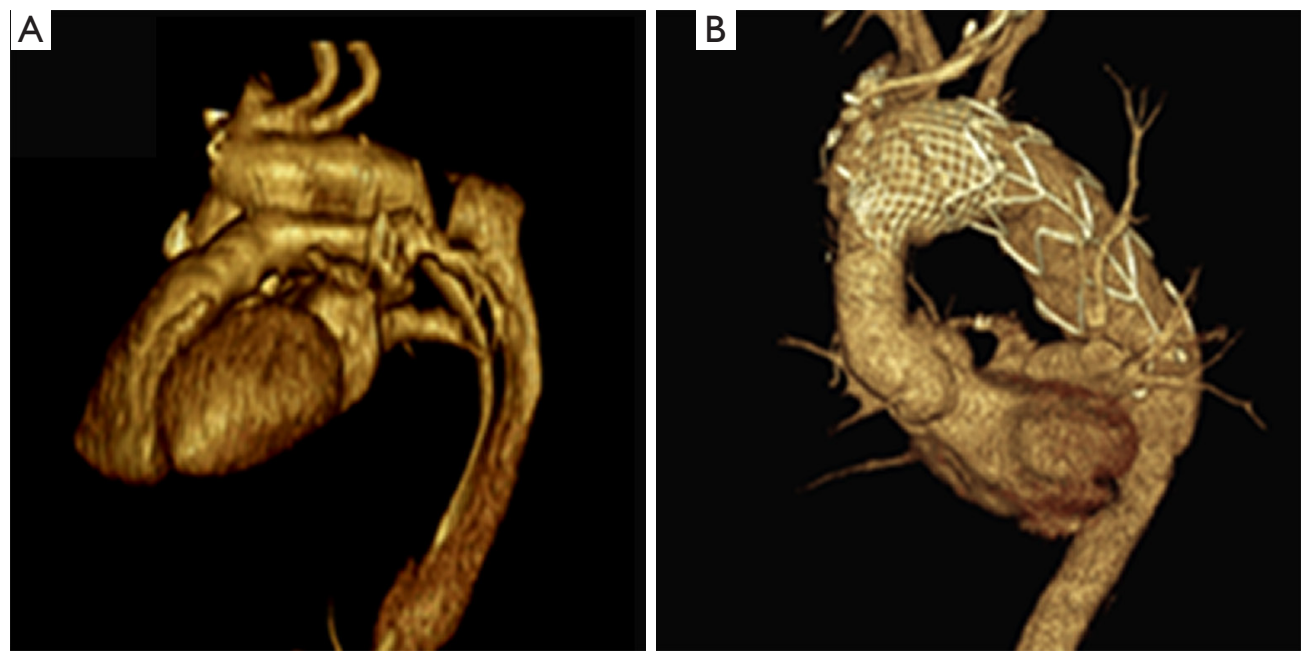

Figure 4 Patient control after combined treatment of aortic arch and descending thoracic aorta by CT scan. (A) Preoperative 3D volume rendering reconstruction of dissected aortic arch; (B) 3D volume rendering reconstruction of implanted Djumbodies Dissection System with associated endovascular stent grafting of the descending thoracic aorta.

independent predictor of an increase in aortic diameter of $10 \mathrm{~mm} /$ year or more. More than $50 \%$ of patients with patent false lumen and aortic arch or descending thoracic aortic diameter greater than $40 \mathrm{~mm}$ show a progressive and significant increase in maximum diameter of the distal aorta (13). Marfan syndrome, postoperative descending aortic diameter greater than $40 \mathrm{~mm}$ and a true lumen smaller than $25 \%$ of the total lumen were found to be other 
Table 4 Late results

\begin{tabular}{lc}
\hline Variables & No. of patients $(\mathrm{n}=11)(\%)$ \\
\hline Mortality & $1(9.0)$ \\
Aortic related & 0 \\
Aortic rupture; $\mathrm{n}(\%)$ & 0 \\
Type I B endoleak; $\mathrm{n}(\%)$ & $2(18.2)$ \\
Type A aortic dissection; $\mathrm{n}(\%)$ & 0 \\
Major complications; $\mathrm{n}(\%)$ & 0 \\
Thoracic Aortic enlargement & $3(27.3)$ \\
LSA related symptoms & 0 \\
False lumen thrombosis & $2(18.2)$ \\
False lumen partial thrombosis & $3(27.3)$ \\
Secondary operation & \\
Open surgery; $\mathrm{n}(\%)$ & $1(9.0)$ \\
Secondary TEVAR; $\mathrm{n}(\%)$ & $2(18.2)$ \\
Secondary EVAR; $\mathrm{n}(\%)$ & 0 \\
\hline
\end{tabular}

TEVAR, Thoracic Endovascular Aortic Repair; LSA, left subclavian artery.

independent predictors of progressive dilation of distal thoracic aorta and aorta-related reoperation $(7,13,16)$. Conversely the closure of the intimal tear and thrombosis of the false lumen at the first operation have a protective impact on late aortic dilatation and rupture reducing rate of aortic related operation $(13,17)$. When emergency ascending aorta and hemiarch replacement is performed without total aortic arch replacement the reported false lumen patency of the downstream aorta ranged from $43 \%$ to $77.5 \%$. Although patency of false lumen was lower in patients with total arch replacement though the difference was not statistically significant $(7,12,18)$.

Indications for reoperation on distal aorta are a diameter of aortic arch or descending thoracic aorta of $55 \mathrm{~mm}$ or more, a rapid aortic growth $(10 \mathrm{~mm} / \mathrm{y})$ and a rupture or impending rupture or signs of visceral malperfusion (12,14-19). During follow-up, $12 \%$ to $24 \%$ of patients with a patent false lumen died because of acute aortic rupture $(6,7,14,20)$ whereas $22.9 \%$ required an aortic-related reoperation often in emergency at a mean time of about 5 years $(12,13)$. These patients underwent reoperation for treatment of aortic arch enlargement or rupture. Reoperation of the aortic arch, especially in patients with previous proximal aortic surgery, is one of the most complicated and technically demanding operations and requires a surgical team with expertise and experience.

As previously mentioned a postoperative descending aortic diameter greater than $40 \mathrm{~mm}$ with a large false lumen is a predictor of distal aortic dilatation or aorta-related events, so it is difficult to establish the best time to perform distal re-intervention in asymptomatic patients.

Conventional open surgery repair remains the gold standard option for the secondary treatment of aortic enlargement or rupture of the downstream aorta regardless of the anatomic location of the aneurysm $(12,20)$. Unfortunately, reoperation is complicated by high morbidity and mortality rates compared with primary surgery especially when the aortic arch is involved $(12,20)$ Currently with the development of the endovascular therapy, new technological solutions can be used to replace the total arch and prevent false lumen patency after surgery for acute TAAD like the "frozen elephant trunk" (FET procedure) $(21,22)$. Although spinal cord ischemia is considered one of the most serious complications associated with FET, its incidence is low ranging from $1.4 \%$ to $2.0 \%(21,22)$. Some groups recently reported excellent results without increased inhospital mortality and morbidity with the FET procedure for aortic arch replacement compared with conventional surgery $(21,22)$.

Endovascular treatment (TEVAR) was reserved for distal aortic arch, descending or abdominal aorta if the intimal tear is expected to be covered with a stent graft. An option for aortic arch treatment can be a hybrid endovascular approach with surgical transposition of supra-aortic trunks and subsequent endovascular treatment implanting a conventional covered stent-graft in the aortic arch up to ascending graft $(23,24)$.

To overcome these issues, many efforts have been made to obtain a purely endovascular approach of the residual TAAD. Specifically designed to treat residual TAAD or type $\mathrm{B}$ dissection with retrograde extension, use of metal uncovered bare stents might be the ideal solution. Compressing the false lumen, these stents prevent aortic dilatation and preserve blood flow into the supra-aortic vessels, overcoming one of the principal limitations of TEVAR with covered stent graft.

After first reports of the use of metal bare stent $(9,25)$ distally to implanted thoracic endovascular stent graft in order to induce positive aortic remodeling with true lumen expansion and false lumen thrombosis as well as preservation of the visceral arteries in patients with clinical evidence of malperfusion and/or radiologic evidence of complete true lumen collapse, the main currently indications of their use 
is the PETTICOAT approach (9).

In 2002, Roux and coworkers (26) reported the first two deployments in aortic arch of an uncovered steel stent during conventional open surgery for ascending aorta replacement in order to avoid aortic enlargement and further aorta-related reoperation. Other authors reported the use of the Djumbodis device deployed intraoperatively in an antegrade fashion with both favorable and less favorable results $(27,28)$. Unsatisfactory results can be related to Djumbodis System configuration that is correctly performed on a "working aorta" whereas the implant during open surgery procedure is carried out on an "empty aorta". The device is fixed to a deflate aortic wall and is not able to adapt to the systolic and diastolic wall-motion changes during the cardiac cycle after resuming pulsatile perfusion probably invalidating the procedure.

Since 2009 the uncovered stent-grafts have been introduced via open transfemoral access for purely endovascular treatment of residual TAAD as a proximal PETTICOAT approach in aortic arch. This approach avoids: surgical risk related to sterna reentry to perform arch debranching, cardiopulmonary bypass with deep hypothermic circulatory arrest ensuring optimal cerebral and visceral perfusion, true lumen restoration and false lumen reduction, exclusion or obliteration, obtaining an optimal cerebral and visceral perfusion. The main limitation seems to be the indications to only treat slightly enlarged aortic arch with a diameter of less than 40 or $45 \mathrm{~mm}$ and a prevailing patent false lumen. As well in not very enlarged aorta, these devices compressing and/or occluding the proximal false lumen is however useful to successfully avoid progressive thoracic aortic dilatation.

In our study, though of eleven patients, there is no differences for early and late results between patients with stainless steel balloon-expandable devices (DDS system) and those with nitinol self-expandable stents (Jotec E-XL). The main limitation of the first device seems to be its nonself-expanding capability which requires deep systemic hypotension and/or rapid pacing. It does not affect the results and allows to extend the stent to just over $45 \mathrm{~mm}$. The limitation of the nitinol bare stents seems to be the reduced stent diameter when opened of just over $40 \mathrm{~mm}$.

Like endovascular treatment with stent-graft, reserved only for distal aortic arch or descending thoracic aorta, endovascular treatment with bare-stent compared with conventional open surgery, is a less invasive option, associated with fewer postoperative complications, leading to a shortened hospital stay (28).

\section{Conclusions}

Although conventional surgery is still the gold standard for treatment of residual aortic dissection after ascending aorta replacement, Endovascular approach with uncovered metalbare stent is surely an evolving strategy to perform a purely endovascular treatment, especially in high risk patients. Compressing the false lumen, as a proximal PETTICOAT approach of the aortic arch, these stents prevent aortic dilatation and preserve blood flow into the supra-aortic vessels, overcoming one of the principal limitations of TEVAR with covered stent graft. Indicated only for treatment of an aortic arch with a diameter of less than 40 or $45 \mathrm{~mm}$, these devices is however useful to successfully avoid progressive thoracic aortic dilatation, since as previously reported an aortic arch diameter greater than $40 \mathrm{~mm}$ associated with a postoperative patent false lumen is already an important risk factors for aortic enlargement and or rupture.

At present, this purely endovascular approach remains challenging. Obviously, a significantly larger number of treated cases and clinical outcome of these patients at long term follow-up is mandatory to analyze the real efficacy of this procedure.

\section{Acknowledgements}

None.

\section{Footnote}

Conflicts of Interest: The authors have no conflicts of interest to declare.

Ethical Statement: The study was approved by members of the ethics committee of the Hospital "Federico II" of University "Federico II" of Naples Italy, where patients had been hospitalized, with protocol no. 189/2016. Written informed consent was obtained from the parents to publish this manuscript and any accompanying images.

\section{References}

1. Trimarchi S, Nienaber CA, Rampoldi V, et al. Contemporary results of surgery in acute type A aortic dissection: the IRAD experience. J Thorac Cardiovasc Surg 2005;129:112-22. 
2. Auer J, Berent R, Eber B. Aortic Dissection: Incidence, Natural History and Impact of Surgery. J Clin Basic Cardiol 2000;3:151-4.

3. Berretta P, Patel HJ, Gleason TG, et al. IRAD experience on surgical type A acute dissection patients: results and predictors of mortality. Ann Cardiothorac Surg 2016;5:346-51.

4. Conzelmann LO, Weigang E, Mehlhorn U, et al. Mortality in patients with acute aortic dissection type A: analysis of pre- and intraoperative risk factors from the German Registry for Acute Aortic Dissection Type A (GERAADA). Eur J Cardiothorac Surg 2016;49:e44-52.

5. Omura A, Miyahara S, Yamanaka K, et al. Early and late outcomes of repaired acute DeBakey type I aortic dissection after graft replacement. J Thorac Cardiovasc Surg 2016;151:341-8.

6. Fattouch K, Sampognaro R, Navarra E, et al. Longterm results after repair of type a acute aortic dissection according to false lumen patency. Ann Thorac Surg 2009;88:1244-50.

7. Halstead JC, MeierM, Etz C, Spielvogel D, et al. The fate of the distal aorta after repair of acute type A aortic dissection. J Thorac Cardiovasc Surg 2007;133:127-35.

8. Zimpfer D, Czerny M, Kettenbach J, et al. Treatment of acute type a dissection by percutaneous endovascular stentgraft placement. Ann Thorac Surg 2006;82:747-9.

9. Nienaber CA, Kische S, Zeller T, et al. Provisional extension to induce complete attachment after stent-graft placement in type B aortic dissection: the PETTICOAT concept. J Endovasc Ther 2006;13:738-46.

10. Iannelli G, Di Tommaso L, Cirillo P, et al. Treatment of residual type A aortic dissection with implantation of the Djumbodis system: is purely endovascular treatment becoming a reality? J Endovasc Ther 2011;18:368-73.

11. Heinemann M, Laas J, Karck M, et al. Thoracic aortic aneurysms after acute type A aortic dissection: necessity for follow-up. Ann Thorac Surg 1990;49:580-4.

12. Kimura N, Itoh S, Yuri K, et al. Reoperation for enlargement of the distal aorta after initial surgery for acute type A aortic dissection. J Thorac Cardiovasc Surg 2015;149:S91-8.e1.

13. Leontyev S, Haag F, Davierwala PM, et al. Postoperative Changes in the Distal Residual Aorta after Surgery for Acute Type A Aortic Dissection: Impact of False Lumen Patency and Size of Descending Aorta. Thorac Cardiovasc Surg 2017;65:90-8.

14. Kimura N, Taruka M, Kawahito K, et al. Influence of patent false lumen on long term outcome after surgery for acute type A aortic dissection. J Thorac Cardiovasc Surg 2008;136:1160-6.

15. Concistrè G, Casali G, Santaniello E, et al. Reoperation after surgical correction of acute type A aortic dissection: risk factor analysis. Ann Thorac Surg 2012;93:450-5.

16. Song JM, Kim SD, Kim JH, et al. Long-termpredictors of descending aorta aneurysmal change in patients with aortic dissection. J Am Coll Cardiol 2007;50:799-804.

17. Hirotani T, Nakamichi T, Munakata M, et al. Routine extended graft replacement for an acute type A aortic dissection and the patency of the residual false channel. Ann Thorac Surg 2003;76:1957-61.

18. Kim JB, Chung CH, Moon DH, et al. Total arch repair versus hemiarch repair in the management of acute DeBakey type I aortic dissection. Eur J Cardiothorac Surg 2011;40:881-7.

19. Svensson LG, Kouchoukos NT, Miller DC, et al. Expert consensus document on the treatment of descending thoracic aortic disease using endovascular stent-grafts. Ann Thorac Surg 2008;85:S1-41.

20. Olsson C, Hillebrant CG, Liska J, et al. Mortality and reoperations in survivors operated on for acute type $\mathrm{A}$ aortic dissection and implications for catheter-based or hybrid interventions. J Vasc Surg 2013;58:333-9.e1.

21. Tsagakis K, Pacini D, Di Bartolomeo R, et al. Arch replacement and downstream stent grafting in complex aortic dissection: first results of an international registry. Eur J Cardiothorac Surg 2011;39:87-93; discussion 93-4.

22. Di Bartolomeo R, Pacini D, Savini C, et al. Complex thoracic aortic disease: single-stage procedure with the frozen elephant trunk technique. J Thorac Cardiovasc Surg 2010;140:S81-5.

23. Iannelli G, Monaco M, Di Tommaso L, et al. Complicated acute type $\mathrm{B}$ aortic dissection involving the arch: treatment by simultaneous hybrid approach under local anesthesia. J Thorac Cardiovasc Surg 2008;135:1380-2.

24. Czerny M, Funovics M, Schoder M, et al. Transposition of the supra-aortic vessels before stent grafting the aortic arch and descending aorta. J Thorac Cardiovasc Surg 2013;145:S91-7.

25. Kische S, D'Ancona G, Belu IC, et al. Perioperative and mid-term results of endovascular management of complicated type B aortic dissection using a proximal thoracic endoprosthesis and selective distal bare stenting. Eur J Cardiothorac Surg 2015;48:e77-84.

26. Roux D, Brouchet L, Concina P, et al. Type-A acute dissection: combined operation plus stent management. 
Ann Thorac Surg 2002;73:1616-8.

27. Vallabhajosyula P, Szeto WY, Pulsipher A, et al. Antegrade thoracic stent grafting during repair of acute Debakey type I dissection promotes distal aortic remodeling and reduces late open distal reoperation rate. J Thorac Cardiovasc Surg
2014;147:942-8.

28. Caus T, Sirota D, Nader J, et al. Associated bare stenting of distal aorta with a Djumbodis(®) system versus conventional surgery in type A aortic dissection. Ann Cardiothorac Surg 2016;5:336-45.

Cite this article as: Di Tommaso L, Giordano R, Di Tommaso E, Di Palo G, Iannelli G. Treatment with transfemoral baremetal stent of residual aortic arch dissection after surgical repair of acute type an aortic dissection. J Thorac Dis 2018;10(11):6097-6106. doi: 10.21037/jtd.2018.10.51 